\title{
Imaging analytical studies of Old Master paints using FTIR, SIMS and SEMEDX of embedded paint cross sections.
}

\author{
Jaap Boon*, Ester S.B.Ferreira* and Katrien Keune* \\ *Molecular Paintings Research Group, FOM Institute AMOLF, Kruislaan 407, 1098 SJ, \\ Amsterdam, Netherlands. boon@amolf.nl
}

The composition of paint layers in paintings is a challenge for the microscopic analyst of paintings. In comparison to modern composites, the original materials in paintings no longer exist and analytical methods have to be adapted to meet the challenges of the small size of the available sample, the variable oxidation state and the difficulties of separating oxidised condensation polymers and inorganic fractions in the paint. The paint layer structure imposes its own complexities since each layer has unique properties: variable thickness, mixed mineral phases in different particle size distributions, and unique binding medium signatures. Thus, the combined application of spatially resolved analytical techniques using imaging FTIR (FT-6000 Stingray, Digilabs Inc), SEMEDX (FEI XL30 SFEG with EDAX EDX analysis) and static SIMS ( TRIFT II; Phy Electronics Inc) is our preferred approach [1]. The quality of the surface is of crucial importance to obtain any results. In our hands this is accomplished first by wet grinding to thin the embedding material around a paint chip and subsequently dry polishing with an in-house designed sample holder that can feed in micrometer steps using Micromesh ${ }^{\circledR}$ cloths up to 12000 mesh. Under these conditions FTIR imaging presents the chemical functional group distribution, while SIMS gives information about the nature of fatty acids, fatty acyl moieties, metal carboxylates and proteins. Both SIMS and SEMEDX mapping give complimentary elemental information. An example is presented.

The ultramarine colored paint (made from "lapis lazuli") from the painting "The Descent from the Cross" by Rogier van der Weyden (1400-1464) was studied earlier with a focus on the distribution of oil derived constituents in the paint cross section using imaging SIMS and FTIR [1]. An important remaining problem was the composition of a few several micron thick layers just visible by light microscopy. These layers are part of the preparatory process of the painting and could contain evidence for the methodology of underdrawing and imprematura. The layers were too thin for imaging FTIR. SIMS maps demonstrate the presence of lead, calcium and fatty acids (C16FA and C18FA imaged as negative ions) in this layer.

We report here SEMEDX data at 2500x using a $10 \mathrm{kV}$ acceleration voltage to limit the elemental information to the more surfacial layers. A BSE map and element maps of lead, calcium, and carbon are presented in (Fig 1 A-D). No data could be obtained for nitrogen, a potential component of an proteinaceous emulsion paint.

The preparatory layer $\mathrm{b}$ is highlighted in yellow in Fig 1 . Highly reflecting particles in the BSE map represent lead white. Calcium carbonate and azurite (copperhydroxycarbonate) have lower electron reflectivities. Calcium (1C) is mainly present in the chalk ground (layer c) with some individual particles in layer b. Azurite is only present in layer a (see the dark spots upper left in the lead distribution). The lead map (1D) matches the 
lumpy lead white crystal aggregates in layer $a$, but lead is also present in layer $b$ in much smaller crystals and dispersed. Carbon (1B) is more intense in layer b. The carbon X-ray yields from the carbonate containing lead white, azurite and calcium carbonate are much lower. The hot spots in layer $b$ are carbon particles (no BSE reflectivity) assumed to be associated with the underdrawing. If the drawing using a carbon rich medium would have been made on top of layer b, the carbon particles have sunken in. A key argument for a medium rich preparatory layer $\mathrm{b}$ is the much lower relative abundance of pigment particles. Since SIMS indicates fatty acids in this layer, we assume that the carbon distribution in 1B supports the idea of a fatty binding medium. The dispersed lead could stabilize the fatty acids in the form of lead soaps. A crucial question presently under study is whether proteins have been used to formulate this paint layer or if it was a lead enriched oil paint gel. This will be done with imaging SIMS under high magnification.

[1] K. Keune and J.J. Boon (2004). Anal Chem 76, 1374-1385.

[2] We thank Prof. Dr. J.R.J van Asperen de Boer for providing the paint sample (coded A166/b). This research is supported by FOM program 49 and NWO De Mayerne Program (project MOLMAP).

Fig 1 A-D. BSE (1A) and SEMEDX maps of carbon (B), calcium (C) and lead (1D) from a cross section of the ultramarine paint from the robe of Maria in Rogier van der Weyden's Descent from the Cross (Museo del Prado, Madrid). Magnification 2500x.
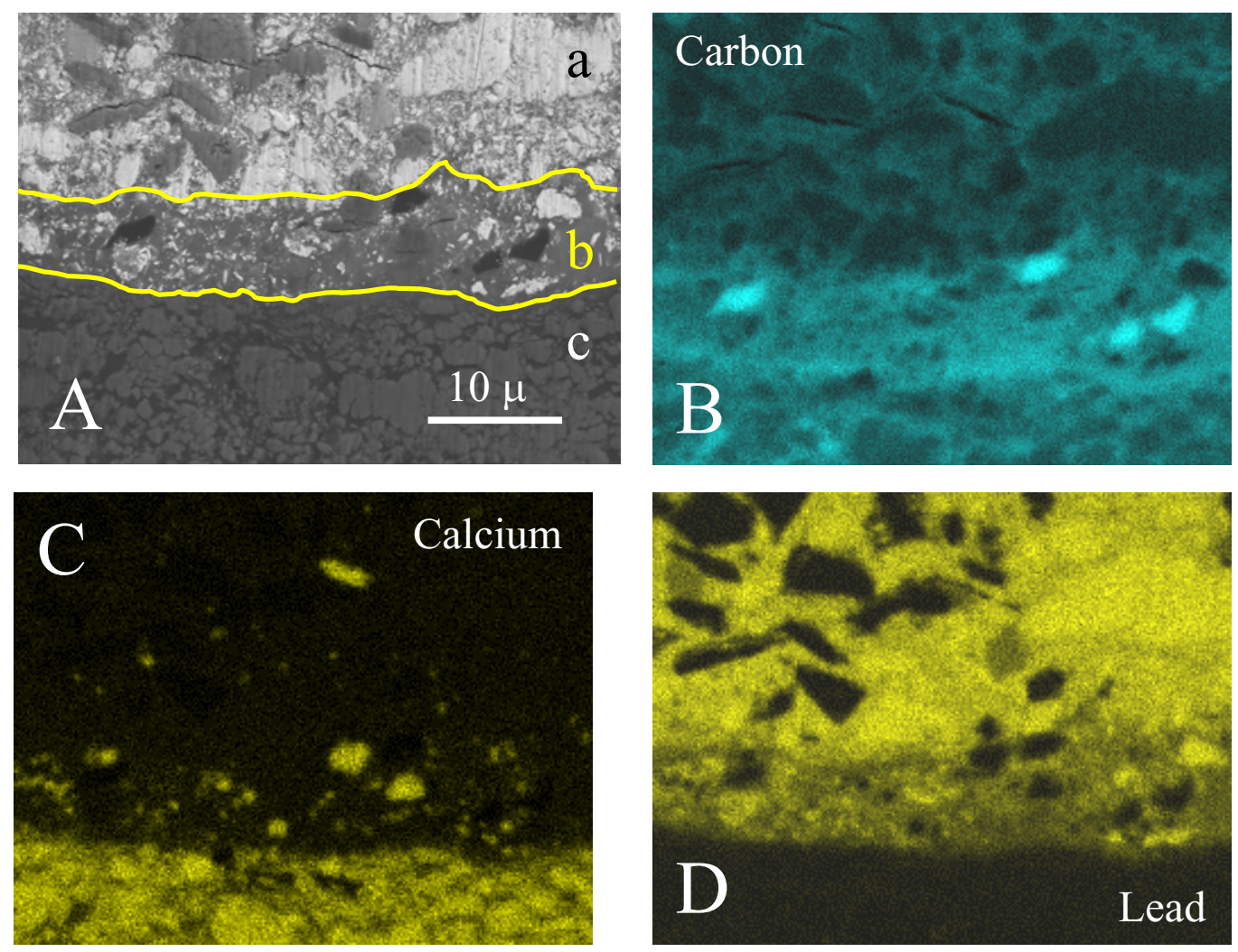